\begin{tabular}{|c|l|}
\hline Title & Shuttle instability induced by an ac gate in a nanoelectromechanical single electron transistor \\
\hline Author(s) & Nishiguchi, Norihiko \\
\hline Citation & $\begin{array}{l}\text { Physical Review B, 78(8),085407 } \\
\text { https://doi.org/10.1103/PhysRevB.78.085407 }\end{array}$ \\
\hline Issue Date & 2008.08 \\
\hline Doc URL & http://hdl.handle.net/2115/34789 \\
\hline Rights & ○ 2008 A merican Physical Society \\
\hline Type & article \\
\hline File Information & GetPDFServlet.pdf \\
\hline
\end{tabular}

Instructions for use 


\title{
Shuttle instability induced by an ac gate in a nanoelectromechanical single-electron transistor
}

\author{
Norihiko Nishiguchi* \\ Division of Applied Physics, Graduate School of Engineering, Hokkaido University, Sapporo, 060-8628, Japan
}

(Received 19 February 2008; revised manuscript received 20 May 2008; published 6 August 2008)

\begin{abstract}
We theoretically investigate shuttle instability induced by an ac gate in a nanoelectromechanical singleelectron transistor containing a moveable grain. The ac gate causes periodic variations of charge on the grain, which turn (in the bias field) to driving force of grain vibrations. The forced grain vibrations and related electron transport show drastic and hysteretic changes for variations of the bias or the gate frequency due to transitions in transport mechanisms between tunneling and shuttling.
\end{abstract}

DOI: 10.1103/PhysRevB.78.085407

PACS number(s): 07.10.Cm, 85.35.Gv, 85.85.+j

\section{INTRODUCTION}

In the last decade, the combination of electron transport and dynamics of nanoscale structures has led to the development of a variety of mass and position detection methods in the quantum limit, ${ }^{1-4}$ to quantum computation, ${ }^{5}$ and to nanoelectronics based on single-electron transport. ${ }^{6,7}$ One of the pioneering studies of such a nanoelectromechanical system was described by Gorelik et al. ${ }^{6}$ Considering a singleelectron transistor (SET) containing a moveable grain, they suggested the shuttle mechanism in which self-sustained vibrations of the grain carry charges between the electrodes. The shuttle mechanism works when an electromechanical instability of the grain position (termed as the shuttle instability) sets in for given strengths of the bias and coupling to a heat bath. ${ }^{6-8}$ Otherwise the grain remains around the electromechanically stable position and tunneling is the dominant transport mechanism as in a conventional SET. Because of the distinct difference in the transport mechanisms, the nanoelectromechanical single-electron transistor (NEMSET) has transport characteristics peculiar to these shuttle and tunnel regimes. Switching between the two regimes at will allows one to exploit the different transport characteristics on a single device, opening up a wealth of applications for NEM-SET in nanoelectronics.

We found in a previous work ${ }^{9}$ that a large dc gate voltage halts the grain vibrations of the NEM-SET and then the dominant transport mechanism changes from shuttling to tunneling. The shuttle mechanism reversibly recovers after turning off the dc gate. This switching mechanism is passive since the dc gate simply regulates energy pumping from the bias field to the grain vibrations; the dc gate cannot therefore excite the grain vibrations in the tunnel regime.

In contrast to the dc gate, an ac gate is able to drive grain vibrations in the tunnel regime because periodic changes in the number of electrons on the grain caused by the ac gate convert, in the bias field, to alternate electrostatic force that drives the grain vibrations. The electrostatic force is actually composed of not only a periodic component with the gate frequency but also a random one due to stochastic electron tunneling. The latter causes intrinsic damping of grain vibrations ${ }^{10}$ however, the former plays a dominant role in driving the grain vibrations. Provided that the forced grain vibrations trigger the shuttle mechanism, the current will be proportional to the gate frequency and then the current is controllable through the tunable gate frequency. In addition, the current noise is expected to be low. ${ }^{8,11-13}$ In spite of these important advances in the field, the induction of shuttle instability by the ac gate and change in the related current and noise properties are not well understood. In the present study, we investigate theoretically the induction of the shuttle instability by the ac gate and related transport properties in a classical NEM-SET.

The present paper is planned as follows: In Sec. II, a model of the NEM-SET is given. We also give in this section the formalism for electron tunneling and grain dynamics. Considering two extreme cases of grain vibrations, we investigate the transitions between shuttling and tunneling in Sec. III. In Sec. IV, we illustrate that the transitions in the grain dynamics take place for variations of bias or of gate frequency. Changes in current and noise related to the transitions are investigated in Sec. V. A summary is given in Sec. VI.

\section{MODEL AND FORMALISM}

The NEM-SET under consideration contains a classical grain of mass $m$ confined in a harmonic potential of frequency $\omega_{0}$ (Fig. 1). The Hamiltonian of the grain reads

$$
H_{v, n}=\frac{p^{2}}{2 m}+\frac{m \omega_{0}^{2}}{2} x^{2}+(-e) x E_{b} n,
$$

where $E_{b}(>0)$ is the bias electric field, $e(>0)$ is the elementary charge, and $n$ is the excess number of electrons on the grain with respect to the electrically neutral state. Electron transport in the NEM-SET is owing to single-electron tunneling and $n$ changes by one for each tunneling event. We investigate the case in which only the two states with $n=0$ and 1 are involved in transport at $T=0 \mathrm{~K}$.

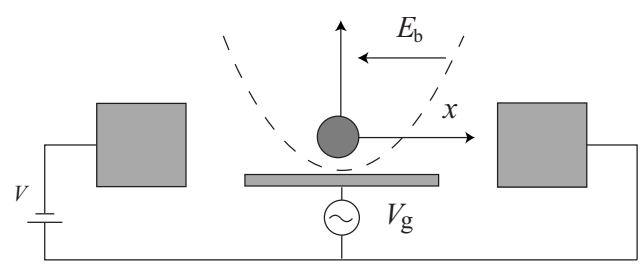

FIG. 1. Model of a NEM-SET. The dashed curve indicates the harmonic confining potential of the grain. 
We are interested in current and noise properties related to the grain dynamics. The transport properties can be derived from the number of charges transferred to the drain ${ }^{14}$ and then we introduce the probability $\mathcal{P}^{(N)}(t)$ that $N$ electrons passed to the drain, where the tunneling begins at $t=0$. The probability also depends on the grain position $x$, momentum $p$, and the number $n$ of electrons on the grain and the change of the $N$-resolved subprobability distribution $\mathcal{P}_{n}^{(N)}(x, p, t)$ is described by the following Klein-Kramers equation ${ }^{15}$ containing tunneling terms:

$$
\begin{aligned}
\frac{\partial}{\partial t} \mathcal{P}_{0}^{(N)}(x, p, t)= & \left\{H_{v, 0}, \mathcal{P}_{0}^{(N)}(x, p, t)\right\}+i \mathcal{L}_{\mathrm{diss}} \mathcal{P}_{0}^{(N)}(x, p, t) \\
& -\Gamma_{L} e^{-2 x / \lambda} \mathcal{P}_{0}^{(N)}(x, p, t)+\Gamma_{R} e^{2 x / \lambda} \mathcal{P}_{1}^{(N-1)}(x, p, t), \\
\frac{\partial}{\partial t} \mathcal{P}_{1}^{(N)}(x, p, t)= & \left\{H_{v, 1}, \mathcal{P}_{1}^{(N)}(x, p, t)\right\}+i \mathcal{L}_{\mathrm{diss}} \mathcal{P}_{1}^{(N)}(x, p, t) \\
& +\Gamma_{L} e^{-2 x / \lambda} \mathcal{P}_{0}^{(N)}(x, p, t)-\Gamma_{R} e^{2 x / \lambda} \mathcal{P}_{1}^{(N)}(x, p, t)
\end{aligned}
$$

In Eqs. (2) and (3), $\{\cdot, \cdot\}$ indicates the Poisson bracket and $i \mathcal{L}_{\text {diss }}$ is the Liouville operator representing energy dissipation;

$$
i \mathcal{L}_{\mathrm{diss}}=2 \gamma_{\infty} \frac{\partial}{\partial p} p+D \frac{\partial^{2}}{\partial p^{2}},
$$

where $\gamma_{\infty}$ is the energy dissipation coefficient due to coupling with a heat bath. The diffusion constant $D$ is given by $D=\gamma_{\infty} m k_{B} T$ at temperature $T$ so that the distribution function exhibits the Boltzmann distribution at the thermal equilibrium state. $\Gamma_{L(R)}$ is the tunneling rate between the left (right) lead and the grain, which are given below, and $e^{ \pm 2 x / \lambda}$ is the modulation of the tunneling rates due to the grain motion, where $\lambda$ is a typical length of tunneling.

The subprobability distribution $\mathcal{P}_{n}(x, p, t)$ is given by

$$
\mathcal{P}_{n}(x, p, t)=\sum_{N=0}^{\infty} \mathcal{P}_{n}^{(N)}(x, p, t),
$$

which is useful for taking an average of observable $A(x, p)$ in the phase space as

$$
\langle A\rangle(t)=\sum_{n} \iint A(x, p) \mathcal{P}_{n}(x, p, t) d x d p .
$$

The probability $P_{n}$ that $n$ electrons are on the grain is derived as

$$
P_{n}(t)=\iint \mathcal{P}_{n}(x, p, t) d x d p .
$$

Here we also introduce $S_{n}$ defined by

$$
S_{n}(t)=\iint \sum_{N=1}^{\infty} N \mathcal{P}_{n}^{(N)}(x, p, t) d x d p,
$$

which is useful for evaluating current and noise below.
Taking the average of the grain position and momentum using Eqs. (2), (3), and (6), we have the equation of motion of the grain. We introduce here the dimensionless position $\xi$ in a unit of the characteristic tunneling length $\lambda$; $\langle x\rangle=\lambda\left(\xi+\frac{\kappa}{2}\right)$. Here $\kappa$ is a coupling constant between the electric system and the grain dynamics, which is defined by the ratio of electrostatic force to mechanical restoring force as

$$
\kappa=\frac{e E_{b}}{m \omega_{0}^{2} \lambda} .
$$

$\kappa \lambda$ is equal to the static grain position for $n=1$ and the origin of $\xi$ is put at the midpoint between the stable positions for $n=0$ and 1 . The equation of motion of the grain yields, in terms of the dimensionless variables,

$$
\ddot{\xi}+2 \gamma_{\infty} \dot{\xi}+\omega_{0}^{2} \xi=\omega_{0}^{2} \kappa\left(\langle n\rangle-\frac{1}{2}\right) .
$$

The expectation value of $n$ is equal to $P_{1}$, i.e.,

$$
\langle n\rangle=P_{1}
$$

since we consider only the states with $n=0$ and 1 in the present work.

Integrating Eqs. (2) and (3) over the phase space and summing up with respect to $N$, we have the master equation for $P_{n}$. For derivation of equation of motion of $S_{n}$, we multiply Eqs. (2) and (3) by $N$, integrate them in the phase space, and sum up with respect to $N$ in the same way. Although $\mathcal{P}_{n}^{(N)}(x, p, t)$ distributes in the phase space, the distribution has a sharp peak in the phase space at $T=0 \mathrm{~K}$ because the diffusion term disappears. In that case, we may approximate that $e^{ \pm 2 x / \lambda} \approx e^{ \pm(2 \xi+\kappa)}$. Hence $P_{n}$ and $S_{n}$ obey the following master equation with the initial condition $S_{n}(0)=0$ since $\mathcal{P}_{n}^{(N)}(x, p, t=0)=0$ for $N>0$ :

$$
\begin{gathered}
\dot{\mathbf{R}}_{0}=-\Gamma_{L} e^{-2 \xi} \mathbf{R}_{0}+\Gamma_{R} e^{2 \xi}\left[\mathbf{R}_{1}+\left(0, P_{1}\right)^{T}\right], \\
\dot{\mathbf{R}}_{1}=\Gamma_{L} e^{-2 \xi} \mathbf{R}_{0}-\Gamma_{R} e^{2 \xi} \mathbf{R}_{1},
\end{gathered}
$$

where $\mathbf{R}_{n} \equiv\left(P_{n}, S_{n}\right)^{T}$ and we redefine $\Gamma_{L} e^{-\kappa}$ by $\Gamma_{L}$ and $\Gamma_{R} e^{\kappa}$ by $\Gamma_{R}$, respectively.

Based on the orthodox theory, we derive the tunneling rates $\Gamma_{L}$ and $\Gamma_{R}$ as

$$
\begin{gathered}
\Gamma_{R}=\frac{1}{e^{2} R_{T}} F\left(\mathcal{E}+e V_{G}\right), \\
\Gamma_{L}=\frac{1}{e^{2} R_{T}} F\left(e V-\mathcal{E}-e V_{G}\right),
\end{gathered}
$$

where $R_{T}$ is the tunnel resistance between the electrode and the grain for $\xi=0 . \mathcal{E}$ is the charging energy of the system; $\mathcal{E}=\frac{e^{2}}{2 C} . C$ is the capacitance of the system, which is composed of that between the grain and the gate and that between the grain and electrodes. Because of the grain vibrations, the latter depends on the grain position and then the total capacitance has the grain position dependence. The vibration amplitude of the grain will become an order of $\lambda$ since the driving force switches the direction for each electron tunnel- 
ing. $\lambda$ is comparable to or smaller than $1 \AA$ from the work function of metals such as gold. On the other hand, supposing that the gap between the electrodes is comparable to or larger than $1 \mathrm{~nm},{ }^{16}$ the ratio of the vibrational amplitude to the gap between the electrodes is an order of 0.1 at most. Considering that the modulation of the total capacitance $C$ is small, we put $C$ as a constant in this work. In Eqs. (14) and (15), $F(\Delta E)$ yields $F(\Delta E)=\Delta E \Theta(\Delta E)$ at $T=0 \mathrm{~K}$, where $\Theta(x)$ is the Heaviside step function. We set $e V=2 \mathcal{E}$ and assume a sinusoidally varying gate voltage at frequency $\omega_{G}$. The ac gate alternately raises and lowers both the energy levels for $n=0$ and 1, which are, however, limited between the Fermi levels of the source and drain, i.e., $-\mathcal{E} \leq \mathrm{eV}_{G} \leq \mathcal{E}$ to prevent the current from flowing against the voltage bias at $T=0 \mathrm{~K}$. Putting $\gamma=\frac{1}{R_{T} C}$ and introducing the dimensionless gate amplitude $U_{G}(t)=\frac{\mathrm{eV}_{G}(t)}{\mathcal{E}}$, we set $\Gamma_{L, R}$ as

$$
\Gamma_{L, R}=\frac{\gamma}{2}\left[1 \pm U_{G}(t)\right],
$$

where $U_{G}$ is restricted in the region $0 \leq\left|U_{G}\right| \leq 1$. The periodic variations of $\Gamma_{R, L}$ at $\omega_{G}$ induce periodic changes at the same frequency in the number of electrons on the grain, which converts in the bias to electrostatic force driving the grain vibrations.

The current and noise are obtained in terms of $P_{n}$ and $S_{n}$. The expectation value of the total charge $Q$ transferred to the drain within time interval $t$ is given by

$$
\langle\langle Q(t)\rangle\rangle=-e\left[S_{0}(t)+S_{1}(t)\right]
$$

and then the current is estimated as

$$
I=-\lim _{x \rightarrow \infty} \frac{\langle\langle Q(t)\rangle\rangle}{t} .
$$

The zero-frequency noise power spectrum $S(0)$ is derived by using the charge fluctuation $\Delta Q^{2}$ in the large time limit as

$$
S(0)=\lim _{x \rightarrow \infty} \frac{2}{t} \Delta Q^{2}(t)
$$

and the Fano factor $F$ is defined by

$$
F=\frac{S(0)}{2 e I} .
$$

The charge fluctuation $\Delta Q^{2}$ is obtained from the time derivative of $\Delta Q^{2}$ given by

$$
\frac{d}{d t} \Delta Q^{2}=e^{2}\left\{2 \Gamma_{R} e^{2 \xi} S_{1}+\Gamma_{R} e^{2 \xi} P_{1}\left[1-2\left(S_{0}+S_{1}\right)\right]\right\} .
$$

\section{SHUTTLE INSTABILITY}

We derive a balance equation of vibrational energy, with which we investigate occurrence of the shuttle instability. Considering that the grain vibrates at $\omega_{G}$, we express the grain displacement as

$$
\xi=X \sin \omega_{G} t .
$$

Allowing that the amplitude $X$ varies slowly in comparison with $\omega_{G}$ and putting Eq. (22) into Eq. (10), we have

$$
\begin{aligned}
& 2 \omega_{G} \dot{X} \cos \omega_{G} t+2 \gamma_{\infty} \omega_{G} X \cos \omega_{G} t+\left(\omega_{0}^{2}-\omega_{G}^{2}\right) X \sin \omega_{G} t \\
& \quad+2 \gamma_{\infty} \dot{X} \sin \omega_{G} t=\omega_{0}^{2} \kappa\left(P_{1}-\frac{1}{2}\right),
\end{aligned}
$$

where the term containing $\ddot{X}$ is ignored. Multiplying Eq. (23) by $\cos \omega_{G} t$ and integrating over a time interval $\frac{2 \pi}{\omega_{G}}$, we have

$$
2 \omega_{G} \dot{X}=-2 \gamma_{\infty} \omega_{G} X+\omega_{0}^{2} \kappa\left[\frac{\omega_{G}}{\pi} \int_{0}^{2 \pi / \omega_{G}} \cos \omega_{G} t\left(P_{1}-\frac{1}{2}\right) d t\right] .
$$

Here we define the vibration energy $E$ of the grain by the squared velocity as

$$
E=\left(\frac{\omega_{G}}{\omega_{0}}\right)^{2} X^{2}
$$

Multiplying Eq. (24) by $\frac{\omega_{G}}{\omega_{0}^{3}} X$, we obtain the balance equation of $E$;

$$
\frac{1}{\omega_{0}} \dot{E}=-2 \frac{\gamma_{\infty}}{\omega_{0}} E+W(E) .
$$

The first term in the right-hand side of Eq. (26) denotes energy dissipation to the heat bath and the second term denotes the energy pumped from the bias field defined by

$$
W(E)=\frac{\kappa \omega_{G}}{\pi} \int_{0}^{2 \pi / \omega_{G}} \cos \omega_{G} t\left(P_{1}-\frac{1}{2}\right) d t \sqrt{E} .
$$

A steady vibrational state appears when the first term and second one are balanced.

We apply Eq. (26) to two extreme cases: small vibrations and large ones. For $X \ll 1$, the grain motion will affect little occurrence of electron tunneling and then the electron tunneling is stochastic as in a SET containing an immobile grain or the conventional SET. Putting $\xi=0$ and $P_{0}=1-P_{1}$ and solving Eq. (13), $P_{1}$ is obtained as

$$
P_{1}=\frac{1}{2}+U_{G}^{0} \cos \theta_{1} \sin \left(\omega_{G} t+\psi\right) .
$$

$P_{1}$ is in advance of the grain vibrations (22) by $\psi$, which is determined from Eq. (10) as

$$
\psi=\tan ^{-1} \frac{2 \omega_{G} \gamma_{\infty}}{\omega_{0}^{2}-\omega_{G}^{2}} .
$$

For the derivation of $P_{1}, U_{G}$ is put as

$$
U_{G}=U_{G}^{0} \sin \left(\omega_{G} t+\theta_{1}+\psi\right),
$$

where $\theta_{1}$ is a phase difference between the ac gate and $P_{1}$, which is given by

$$
\theta_{1}=\tan ^{-1} \frac{\omega_{G}}{\gamma} .
$$

Substituting Eq. (28) into Eq. (27), we obtain the pumped energy $W_{\text {sto }}$ for stochastic tunneling as 


$$
W_{\text {sto }}=\frac{U_{G}^{0} \gamma}{\sqrt{\omega_{G}^{2}+\gamma^{2}}} \frac{\kappa \gamma_{\infty} \omega_{G} \sqrt{E}}{\sqrt{4 \gamma_{\infty}^{2} \omega_{G}^{2}+\left(\omega_{0}^{2}-\omega_{G}^{2}\right)^{2}}} .
$$

Because $W_{\text {sto }} \propto \sqrt{E}$, there is always a steady vibrational state satisfying Eq. (26) even if $E$ is very small, in contrast to the NEM-SET in the absence of the ac gate, ${ }^{7,9}$ in which $W(E)$ is expanded in a power series of $E$. The vibrational energy satisfying Eq. (26) yields

$$
E_{\text {sto }}=\frac{U_{G}^{0^{2}}}{4} \frac{\gamma^{2}}{\omega_{G}^{2}+\gamma^{2}} \frac{\kappa^{2} \omega_{0}^{2} \omega_{G}^{2}}{4 \gamma_{\infty}^{2} \omega_{G}^{2}+\left(\omega_{0}^{2}-\omega_{G}^{2}\right)^{2}} .
$$

On the other hand, for $X \gg 1$, tunneling will occur quickly at a high probability when the grain approaches the electrodes because of the exponential dependence on $\xi$ in the tunnel rates. Since it is hard to obtain analytically $P_{0}$ and $P_{1}$ from the master equation, we suppose that $P_{0}$ and $P_{1}$ vary complementarily between 0 and 1 like a telegraph signal, considering the coherent tunneling. Using the Fourier transform, we describe $P_{1}$ as

$$
P_{1} \approx \frac{1}{2}+\sum_{n=1}^{\infty} \frac{2}{(2 n-1) \pi} \sin \left[(2 n-1)\left(\omega_{G} t+\psi\right)\right] .
$$

As readily understood from Eq. (27), only the fundamental frequency component of $\omega_{G}$ in Eq. (34) contributes to the integral of pumped energy. Hence, the pumped energy $W_{\text {coh }}$ using Eq. (34) becomes

$$
W_{\mathrm{coh}}=\frac{4}{\pi} \frac{\kappa \omega_{G} \gamma_{\infty} \sqrt{E}}{\sqrt{4 \gamma_{\infty}^{2} \omega_{G}^{2}+\left(\omega_{0}^{2}-\omega_{G}^{2}\right)^{2}}},
$$

and the energy at the steady vibrational state yields

$$
E_{\mathrm{coh}}=\frac{4}{\pi^{2}} \frac{\kappa^{2} \omega_{0}^{2} \omega_{G}^{2}}{4 \gamma_{\infty}^{2} \omega_{G}^{2}+\left(\omega_{0}^{2}-\omega_{G}^{2}\right)^{2}} .
$$

Supposing that the grain carries electrons between the electrodes like shuttling, the phase $\psi$ should be ideally $\frac{\pi}{2}$ from Eqs. (22) and (34). On the other hand, $\psi$ becomes $\frac{\pi}{2}$ when $\omega_{G}=\omega_{0}$ from the gate frequency dependence of $\psi$ [Eq. (29)]. The amplitude $X$ becomes large at the gate frequency because the grain vibrations resonate as we presupposed that $X \gg 1$ for the coherent tunneling. Hence, the shuttling induced by the ac gate is expected to occur when the grain vibrations resonate.

It is obvious mathematically that $W_{\text {sto }}$ is always smaller than $W_{\text {coh }}$ since $0<U_{G}^{0} \leq 1$ and $\frac{\gamma}{\sqrt{\omega_{G}^{2}+\gamma^{2}}}<1$. The difference in magnitude of $W$ stems from that the energy pumping in the latter is more effective than the former because the electrostatic force pushing the charged grain is almost constant in proportion to $\langle n\rangle$ in the middle region between the electrodes in the latter, whereas the force changes in phase with the grain motion in the former. As $X$ increases, the charge on the grain or the force acting on the grain will switch gradually from simple sinusoidal variations to variations similar to a telegraph signal. Accordingly, the pumped energy $W(E)$ becomes larger than $W_{\text {sto }}$ in the intermediate region of $X$ $\approx O(1)$ and transfers to $W_{\text {coh }}$. Supposing a smooth variation of $W(E)$ between $W_{\text {sto }}$ and $W_{\text {coh }}$, we speculate that a line

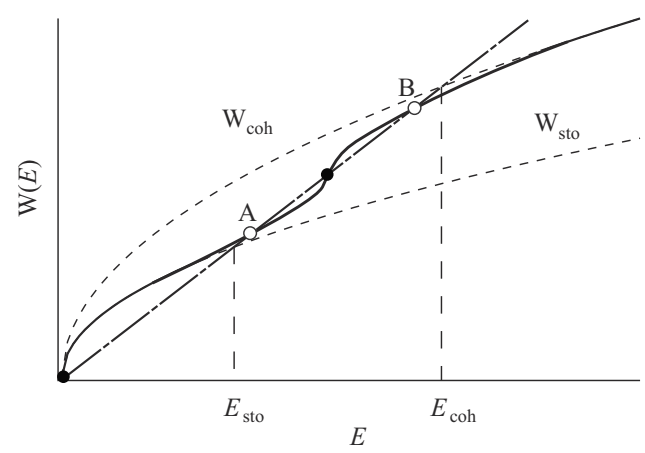

FIG. 2. Schematic of pumped energy $W(E)$ versus vibrational energy $E$. The solid line indicates that $W(E)$ changes from $W_{\text {sto }}$ to $W_{\text {coh }}$ with increasing $E$. Intersections of $W(E)$ with the energy dissipation $2 \frac{\gamma_{\infty}}{\omega_{0}} E$ (dot-long-dashed line) give the solutions of Eq. (26), which are represented by the open and solid circles. $E_{\text {sto }}$ and $E_{\text {coh }}$ indicate the intersections of $W_{\text {sto }}$ and $W_{\text {coh }}$ with $2 \frac{\gamma_{\infty}}{\omega_{0}} E$, respectively.

connecting $W_{\text {sto }}$ and $W_{\text {coh }}$ becomes a step as shown in Fig. 2 . As mentioned above, the steady states appear when $W(E)$ balances with $2 \frac{\gamma_{\infty}}{\omega_{0}} E$. Figure 2 shows a case that $W(E)$ intersects with $2 \frac{\gamma_{\infty}}{\omega_{0}} E$ at four different energies including $E=0$, which are denoted by the open and solid circles. The open circles labeled A and B indicate two steady states, which are stable for fluctuations in $E$, whereas the solid circles indicate unstable states.

As Eqs. (32) and (35) show clearly, $W(E)$ increases in proportion to $\kappa$ in the entire region of $E$. The state $\mathrm{A}$ and the unstable solution between the states A and B get closer and merge in energy as $\kappa$ increases. The state A disappears for further increase in $\kappa$, which causes a transition to the state B. Once the state A disappears, the grain vibration energy begins to increase, whose rate is proportional to the energy difference $W(E)-2 \frac{\gamma_{\infty}}{\omega_{0}} E$. The rate is very low at the onset of the transition since the energy difference is small, but the grain vibrations soon grow rapidly and the amplitude $X$ bursts. The transition completes when the pumped energy is balanced with the energy dissipation and eventually, the vibrational state becomes the state B. Such a transition is also anticipated for variations of the gate frequency since Eqs. (32) and (35) have maximum magnitude at the resonance frequency. On the other hand, the state B disappears with decreasing $\kappa$ or shifting $\omega_{G}$ far from $\omega_{0}$ and then there is only the state $\mathrm{A}$ in that case. The transition from the state $\mathrm{B}$ to state A occurs due to energy dissipation. When there are two steady states as shown in Fig. 2, either of the two states is realized, depending on the preceding state. Hence, the changes of $\kappa$ and/or $\omega_{G}$ cause hysteretic transitions between the states $\mathrm{A}$ and $\mathrm{B}$.

The shuttle instability in the absence of gate means disappearance of the electromechanical stable grain position and the simultaneous transition to self-sustained grain vibrations with coherent tunneling. In this study, we regard the disappearance of the state $\mathrm{A}$ and the transition to the state $\mathrm{B}$ as the shuttle instability in the presence of the ac gate.

We attempt to derive the threshold magnitude of $\kappa$ for the shuttle instability induced by the ac gate, at which the state A and an unstable state coincide in energy as mentioned above. 
As discussed above, $P_{0}$ and $P_{1}$ are almost independent of the amplitude $X$ for $X \ll 1$ and the resultant pumped energy $W(E)$ has the $\sqrt{E}$ dependence. However, $W(E)$ deviates from the $\sqrt{E}$ dependence as the grain vibrations develop since the dependence of $P_{n}$ on $X$ becomes significant. Expanding the probabilities $P_{0}\left(=1-P_{1}\right)$ and $P_{1}$ and the factors $e^{ \pm 2 \xi}$ of the tunneling rates in Eq. (13) into a power series of $X$ as

$$
\begin{gathered}
P_{1}=\frac{1}{2}+R_{0}+R_{1} X+R_{2} X^{2}+\ldots, \\
e^{ \pm 2 \xi}=1 \pm 2 X \sin \omega_{G} t+2 X^{2} \sin ^{2} \omega_{G} t+\ldots,
\end{gathered}
$$

we have the master equations for each order of $X$ as

$$
\begin{gathered}
\dot{R}_{0}=-\gamma R_{0}+\frac{1}{2} \gamma U_{G}, \\
\dot{R}_{1}=-\gamma R_{1}+\gamma\left(2 R_{0} U_{G}-1\right) \sin \omega_{G} t, \\
\dot{R}_{2}=-\gamma R_{2}+\gamma\left(2 R_{1} U_{G} \sin \omega_{G} t-2 R_{0} \sin ^{2} \omega_{G} t\right. \\
\left.+U_{G} \sin ^{2} \omega_{G} t\right),
\end{gathered}
$$

up to the second order of $X$. We put the ac gate as

$$
U_{G}(t)=U_{G}^{0} \sin \left(\omega_{G} t+\psi+\theta\right),
$$

where $\theta$ is the phase difference at $E$ between the ac gate and $P_{1}$, which reduces to $\theta_{1}$ for $E=0$ as discussed in Eqs. (30) and (31) for $W_{\text {sto }}$. The solutions of Eqs. (39)-(41) for $n=0,1$, and 2 yield

$R_{n}(t)=S_{n} \sin \left(\omega_{G} t+\psi\right)+C_{n} \cos \left(\omega_{G} t+\psi\right)+$ harmonic terms,

where the coefficients $C_{n}$ and $S_{n}$ depend on $\psi, \gamma, \omega_{G}$, and $\theta$, which are given in the Appendix. Putting Eq. (43) into Eq. (37), we obtain $P_{1}$;

$$
P_{1}(t)=\frac{1}{2}+P_{1}^{s} \sin \left(\omega_{G} t+\psi\right)+P_{1}^{c} \cos \left(\omega_{G} t+\psi\right),
$$

where

$$
\begin{aligned}
& P_{1}^{s}=S_{0}+\frac{\omega_{0}}{\omega_{G}} S_{1} \sqrt{E}+\left(\frac{\omega_{0}}{\omega_{G}}\right)^{2} S_{2} E, \\
& P_{1}^{c}=C_{0}+\frac{\omega_{0}}{\omega_{G}} C_{1} \sqrt{E}+\left(\frac{\omega_{0}}{\omega_{G}}\right)^{2} C_{2} E .
\end{aligned}
$$

In Eqs. (45) and (46), $X$ is substituted by $\frac{\omega_{0}}{\omega_{G}} \sqrt{E}$. We exclude the harmonic terms in Eq. (44) since the harmonic terms affect the grain dynamics little, in particular, at the resonance $\omega_{G}=\omega_{0}$. Because the phase difference between $\xi$ and $P_{1}$ is $\psi$, $P_{1}^{c}$ must vanish;

$$
P_{1}^{c}=0 .
$$

The phase $\theta$ introduced in Eq. (42) is determined so that Eq. (47) is satisfied at a given $E$. Because $S_{n}$ and $C_{n}$ depend on $\theta$, the coefficients depend on $E$ via $\theta$ (Fig. 3) although we pre-

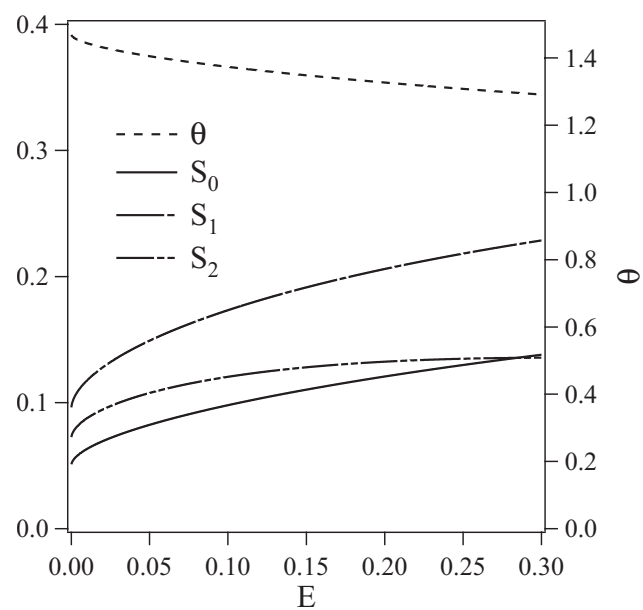

FIG. 3. The $E$ dependence of $\theta, S_{0}, S_{1}$, and $S_{2}$. Used parameters are $U_{G}^{0}=1, \frac{\gamma}{\omega_{0}}=0.1$, and $\omega_{G}=\omega_{0}$.

supposed in Eq. (37) that $R_{n}$ 's are independent of $X$ or $E$. Because of that, tentatively supposing in the following that $S_{n}$ and $C_{n}$ are constant, we continue the analysis.

Substituting Eq. (44) with Eq. (47) into Eq. (27), we obtain $W(E)$ as

$$
W(E)=\kappa\left(A_{0} \sqrt{E}+A_{1} E+A_{2} E^{3 / 2}\right),
$$

where $A_{n}$ is defined by

$$
A_{n}=\left(\frac{\omega_{0}}{\omega_{G}}\right)^{n}\left[\frac{\omega_{G}}{\pi} \int_{0}^{2 \pi / \omega_{G}} \cos \omega_{G} t R_{n} d t\right]=\left(\frac{\omega_{0}}{\omega_{G}}\right)^{n} S_{n} \sin \psi
$$

Putting Eq. (48) into Eq. (26), we have an equation to give energy $E$ at a steady state;

$$
\kappa\left(A_{0} \sqrt{E}+A_{1} E+A_{2} E^{3 / 2}\right)-2 \frac{\gamma_{\infty}}{\omega_{0}} E=0
$$

Solving Eq. (50), we obtain

$$
\sqrt{E_{ \pm}}=\frac{-\left(\kappa A_{1}-2 \frac{\gamma_{\infty}}{\omega_{0}}\right) \pm \sqrt{\left(\kappa A_{1}-2 \frac{\gamma_{\infty}}{\omega_{0}}\right)^{2}-4 \kappa^{2} A_{0} A_{2}}}{2 A_{2}} .
$$

$E_{-}$is the energy of state $\mathrm{A}$ and the state belonging to $E_{+}$is unstable for energy fluctuations as mentioned above. The state A becomes unstable when $E_{-}$and $E_{+}$merge. In other words, $2 \frac{\gamma_{\infty}}{\omega_{0}} E$ becomes a tangent line of $W(E)$. The condition on $\kappa$ that $W(E)$ has the tangent line is

$$
2 \frac{\gamma_{\infty}}{\omega_{0}}-\kappa A_{1}=2 \kappa \sqrt{A_{0} A_{2}}
$$

from which $\kappa$ for the instability is derived as 


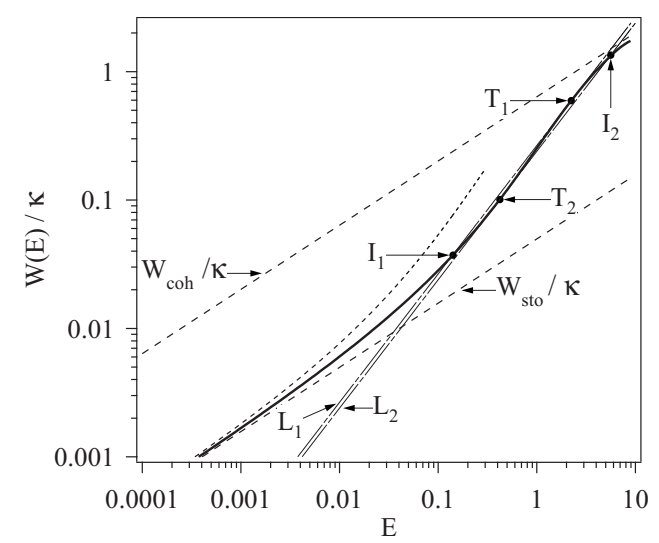

FIG. 4. The log-log plot of $\frac{1}{\kappa} W(E)$ versus $E$ at $\omega_{G}=\omega_{0}$ for $U_{G}^{0}=1$. The dashed lines indicate $\frac{1}{\kappa} W_{\text {sto }}$ and $\frac{1}{\kappa} W_{\text {coh }}$, respectively. $T_{n}$ and $I_{n}(n=1$ and 2) indicate the contact points and intersection points between $\frac{1}{\kappa} W(E)$ and the tangent lines $L_{1}$ and $L_{2}$ (dot-long-dashed lines). The energy at each point is $E_{I_{1}}=0.14$ at $I_{1}$, $E_{T_{1}}=2.24$ at $T_{1}, E_{I_{2}}=5.62$ at $I_{2}$, and $E_{T_{2}}=0.42$ at $T_{2}$.

$$
\kappa_{\text {shuttle }}=2 \frac{\gamma_{\infty}}{\omega_{0}} \frac{1}{A_{1}+2 \sqrt{A_{0} A_{2}}}=2 \frac{\gamma_{\infty}}{\omega_{G}} \frac{1}{S_{1}+2 \sqrt{S_{0} S_{2}}} \frac{1}{\sin \psi} .
$$

The energy $E_{-}$at which the transition from the state A to state B occurs becomes

$$
E_{-}=\left[2 \frac{\gamma_{\infty}}{\omega_{G}} \sqrt{\frac{S_{0}}{S_{2}}} \frac{1}{S_{1}+2 \sqrt{S_{0} S_{2}}}\right]^{2} \frac{1}{\sin ^{2} \psi} .
$$

These results tell that $\kappa_{\text {shuttle }}$ depends on the gate frequency via the phase $\psi$ and becomes minimum when $\psi=\frac{\pi}{2}$ or $\omega_{G}$ $=\omega_{0}$. On the other hand, $\kappa_{\text {shuttle }}$ increases at the off-resonance frequency and the transition is hard to occur.

In order to obtain $\kappa_{\text {shuttle }}$ more quantitatively, we numerically evaluate $W(E)$. We integrate the master equation (13), putting the ac gate as Eq. (42). The phase $\theta$ is adjusted so that the resultant $P_{1}$ contains the only term of $\sin \left(\omega_{G} t+\psi\right)$. Substituting $P_{1}$ into Eq. (27) and numerically integrating, we have $W(E)$. The resultant $\frac{1}{\kappa} W(E)$ is plotted for $U_{G}^{0}=1$, $\frac{\gamma}{\omega_{0}}=0.1$, and $\omega_{G}=\omega_{0}$ with the thick solid line in Fig. 4. The dotted curve in Fig. 4 indicates $\frac{1}{\kappa} W(E)$ estimated from Eq. (48) with the phase $\theta$ adjusted to meet Eq. (47) (Fig. 3) for the same set of parameters. The disagreement in magnitude between Eq. (48) and the numerically obtained $W(E)$ indicates that the excluded terms from Eq. (48) are significant even in the region of $E \ll 0.1$.

Since $\frac{1}{\kappa} W(E)$ is independent of $\kappa$, the appearance and disappearance of steady states depend on the slope of the energy dissipation described by $L=2 \frac{\gamma_{\infty}}{\omega_{0}} \kappa^{-1} E$. As discussed above, $L$ becomes a tangent line of $W(E)$ for the shuttle instability. Two tangent lines, $L_{1}=2 \frac{\gamma_{\infty}}{\omega_{0}} \kappa_{1}^{-1} E$ and $L_{2}=2 \frac{\gamma_{\infty}}{\omega_{0}} \kappa_{2}^{-1} E$, for $W(E)$ are plotted with the dot-long-dashed lines in Fig. 4, whose slopes are $2 \frac{\gamma_{\infty}}{\omega_{0}} \kappa_{1}^{-1}=0.266$ and $2 \frac{\gamma_{\infty}}{\omega_{0}} \kappa_{2}^{-1}=0.239$, respectively. The contact points of $W(E)$ with each line are denoted by the arrows labeled as $T_{1}$ and $T_{2}$ and the intersections by those are

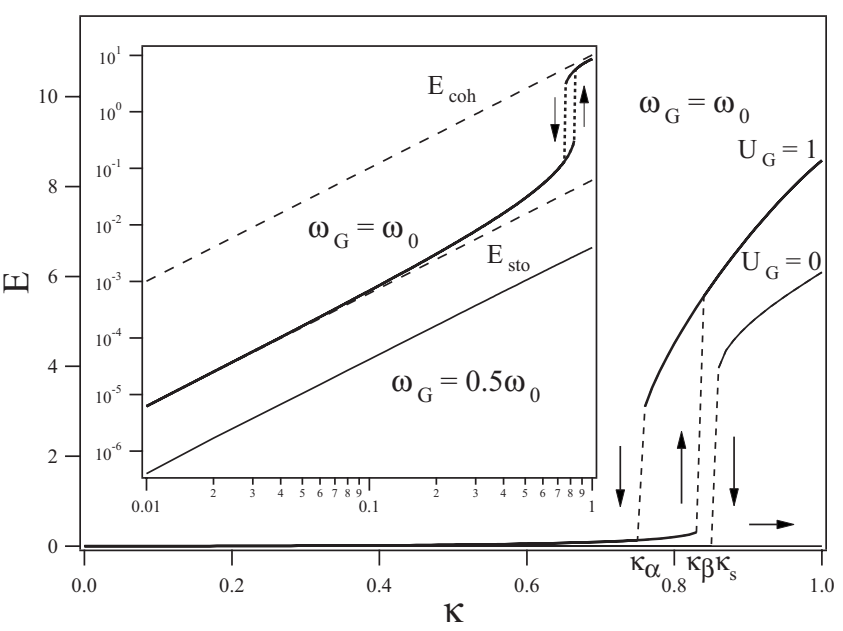

FIG. 5. Vibration energy $E$ versus $\kappa$. The solid lines indicate $E$ in the absence of gate $\left(U_{G}^{0}=0\right)$ and $E$ at $\omega_{G}=\omega_{0}$ for $U_{G}^{0}=1$. The arrows indicate the hysteretic transitions in $E$ for change in $\kappa$. The inset shows the log-log plot of $E$ versus $\kappa$ at $\omega_{G}=\omega_{0}$ and $0.5 \omega_{0}$ for $U_{G}^{0}=1$. The dashed lines indicate $E_{\text {sto }}$ and $E_{\text {coh }}$, respectively. The energies $E$ at the starting points of transition corresponding to $T_{1}$ and $T_{2}$ in Fig. 4 are $E_{T_{1}}=3.1$ and $E_{T_{2}}=0.33$ and those corresponding to $I_{1}$ and $I_{2}$ are $E_{I_{1}}=0.13$ and $E_{I_{2}}=5.56$, respectively.

labeled as $I_{1}$ and $I_{2}$, respectively. The shuttle instability occurs when $T_{2}$ disappears and then $\kappa_{\text {shuttle }}$ becomes $\kappa_{2}$ $=8.368 \frac{\gamma_{\infty}}{\omega_{0}}$. On the other hand, $\kappa$ for transition from $T_{1}$ to $I_{1}$ becomes $\kappa_{1}=7.519 \frac{\gamma_{\infty}}{\omega_{0}}$. These $\kappa$ 's are proportional to $\frac{\gamma_{\infty}}{\omega_{0}}$ and become, for example, $\kappa_{1}=0.752$ and $\kappa_{\text {shuttle }}=\kappa_{2}=0.837$ in case of $\frac{\gamma_{\infty}}{\omega_{0}}=0.1$. The coupling constants $\kappa_{1}$ and $\kappa_{2}$ agree well with those obtained from simulations performed below.

\section{TRANSITIONS BETWEEN VIBRATIONAL STATES}

We confirm the hysteretic transitions in the grain dynamics, solving numerically Eqs. (10), (12), (13), and (16), where we put the ac gate as $U_{G}=U_{G}^{0} \sin \omega_{G} t$ by means of the Runge-Kutta method of fourth order.

To begin with, we investigate the transitions in the absence of gate, i.e., for $U_{G}^{0}=0$. Here $\gamma$ and $\gamma_{\infty}$ are put $\frac{\gamma}{\omega_{0}}=0.1$ and $\frac{\gamma_{\infty}}{\omega_{0}}=0.1$, respectively. Figure 5 shows $E$ versus $\kappa$ for $U_{G}^{0}=0$ and that for $U_{G}^{0}=1$ at $\omega_{G}=\omega_{0}$. For $U_{G}^{0}=0, E$ jumps up from zero to a finite magnitude at $\kappa=1.53$ with increasing $\kappa$ (not shown in Fig. 5). On the other hand, the energy drops off to zero at $\kappa_{s}(=0.86)$ with decreasing $\kappa$, below which no self-sustained vibration occurs and then the NEM-SET is in the tunnel regime.

The transitions induced by the ac gate are confirmed for $U_{G}^{0}=1$ at $\omega_{G}=\omega_{0}$. There is a jump in $E$ at $\kappa_{\beta}(=0.84)$ with increasing $\kappa$ and a drop off in $E$ at $\kappa_{\alpha}(=0.76)$ with decreasing $\kappa$, respectively. We label the characteristic $\kappa$ 's as $\kappa_{\alpha}$ and $\kappa_{\beta}$ to discriminate from $\kappa_{1}$ and $\kappa_{2}$ predicted numerically in Sec. III. It is obvious that $\kappa_{\alpha}$ and $\kappa_{\beta}$ correspond to $\kappa_{1}$ and $\kappa_{2}$, respectively, and they show excellent agreement. Hence, we confirm that $W(E)$ in Fig. 4 provides precise information on the transitions. The hysteretic transitions are also shown on a 


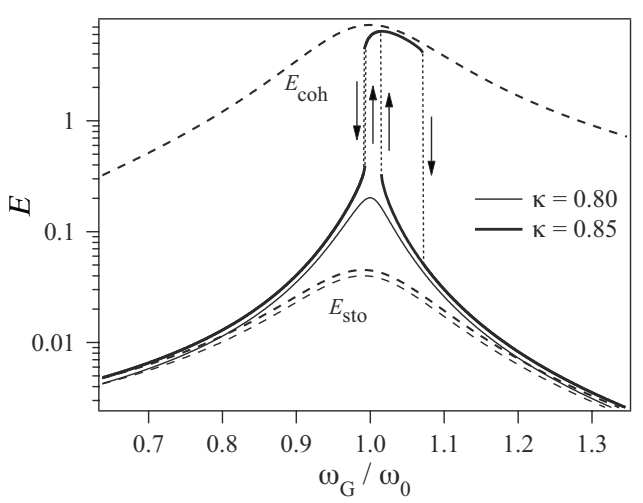

FIG. 6. Vibration energy $E$ versus $\omega_{G}$ for $\kappa=0.80$ (thin line) and 0.85 (thick line). The dashed lines denote $E_{\text {coh }}$ and $E_{\text {sto. }}$ The lower and higher dashed lines of $E_{\text {sto }}$ are estimated for $\kappa=0.80$ and 0.85 , respectively. The arrows indicate the hysteretic transition of $E$ for variation of $\omega_{G}$.

$\log -\log$ plot (solid line) in the inset, where the lower and higher dashed lines indicate $E_{\text {sto }}$ and $E_{\text {coh }}$ for $\omega_{G}=\omega_{0}$, manifesting that the transitions occur between $E_{\text {sto }}$ and $E_{\mathrm{coh}}$. Thus, the ac gate gives rise to the shuttle instability at $\kappa_{\beta}$, which is lower than $\kappa_{s}$ or in the tunnel regime in the absence of gate, and the steady state changes from A to B and vice versa at $\kappa_{\alpha}$.

Another solid line in the inset of Fig. 5 is $E$ versus $\kappa$ for $\omega_{G}=0.5 \omega_{0}$, which agrees completely with $E_{\text {sto }}$ estimated for $\omega_{G}=0.5 \omega_{0}$. It does not show a transition from $E_{\text {sto }}$ to $E_{\text {coh }}$ even if $\kappa>\kappa_{s}$ since energy pumping from the bias is limited because of the off-resonance gate frequency, as understood from Eqs. (32), (35), (53), and (54).

The results for $\omega_{G}=\omega_{0}$ and $0.5 \omega_{0}$ demonstrate that the ac gate not only causes shuttling but also restrains shuttling, depending on the gate frequency. In order to investigate further the gate frequency dependence of the transitions, we plot $E$ versus $\omega_{G}$ for $\kappa=0.8$ and 0.85 around $\omega_{0}$ in Fig. 6 . The dashed lines in Fig. 6 are $E_{\text {sto }}$ and $E_{\text {coh }}$ for $\kappa=0.80$ and 0.85 . $E_{\text {coh }}$ is common to both magnitudes of $\kappa$, whereas the lower and higher dashed lines of $E_{\text {sto }}$ denote the energy for $\kappa=0.8$ and for $\kappa=0.85$, respectively. The hysteretic transitions between $E_{\text {sto }}$ and $E_{\text {coh }}$ occur in the narrow gate frequency region of $\omega_{G} \approx \omega_{0}$ for $\kappa=0.85$, which indicates that $W(E)$ reduces quickly as $\omega_{G}$ moves from $\omega_{0}$ and that it is necessary to raise $\kappa$ to keep shuttling at the off-resonance gate frequency. In contrast to $\kappa=0.85$, the ac gate does not cause a transition to $E_{\text {coh }}$ for $\kappa=0.80$ even at $\omega_{G}=\omega_{0}$, although $E$ becomes five times as large as $E_{\text {sto }}$ at the resonance frequency.

\section{CURRENT AND NOISE PROPERTIES}

The transitions between tunneling and shuttling cause drastic changes in the transport properties. Figure 7 shows the current $I$ and the Fano factor $F$ versus $\omega_{G}$ for $\kappa=0.80$ and 0.85 . For $\kappa=0.85$, the current shows discontinuous and hysteretic changes with respect to $\omega_{G}$ correspondingly to the changes in $E$ in Fig. 6. The current due to shuttling agrees well with the ideal shuttle current

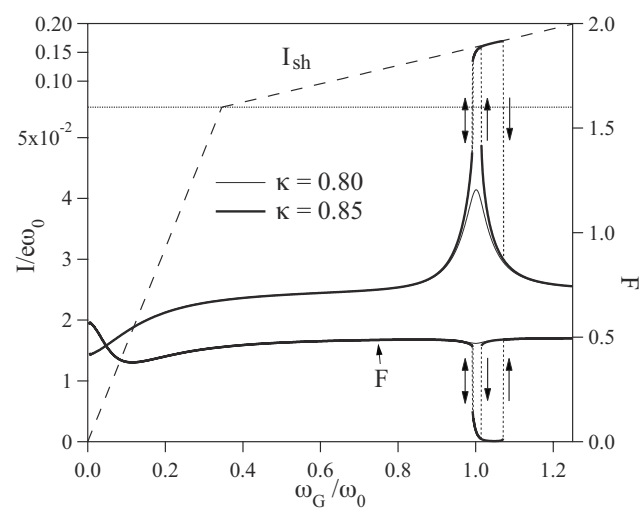

FIG. 7. The current $I$ and the Fano factor $F$ versus $\omega_{G}$ for $\kappa=0.80$ (thin lines) and 0.85 (thick lines). The arrows indicate the hysteretic transitions of $I$ and $F$, corresponding to those in Fig. 6 . The long-dashed line indicates the current $I_{\text {sh}}$.

$$
I_{\mathrm{sh}}=\frac{e \omega_{G}}{2 \pi},
$$

assuming that one electron is transferred within an oscillation of the ac gate and the Fano factor becomes very small because of the coherent tunneling.

In contrast, the current for $\kappa=0.80$ shows a smooth peak at $\omega_{G}=\omega_{0}$, which is brought by the resonance of the grain vibrations. The peak height is, however, much smaller than $I_{\mathrm{sh}}$, which means that electron tunneling does not always take place within every period of grain vibrations, leading to current noise. The Fano factor is approximately 0.5 even at the resonance frequency. The amplitude is at most $X \approx \sqrt{0.2}$ at the resonance frequency from Fig. 6, indicating that the grain motion affects the electron tunneling little. Supposing a static grain at $\xi=0$ in the SET, the Fano factor is given by $F=1$ $-2 \frac{\Gamma_{L} \Gamma_{R}}{\left(\Gamma_{L}+\Gamma_{R}\right)^{2}}$. For a symmetric case, i.e., $\Gamma_{L}=\Gamma_{R}$, the Fano factor becomes $F=\frac{1}{2}$, which is smaller than 1 since electron tunneling is regulated by the occupation of electron on the grain. Hence, the Fano factor for $\kappa=0.8$ indicates that the tunneling remains as stochastic as in the conventional SETs.

\section{SUMMARY}

We theoretically investigated the ac gate effects on the grain dynamics and related electron transport in the NEMSET containing a moveable grain and showed that the shuttle instability is induced by the ac gate. The ac gate affects the electron transport in the device directly and indirectly; the direct effect is to regulate electron tunneling between the electrodes and the grain, which is the same as in the conventional SETs. As a consequence, the charge on the grain changes periodically at the gate frequency, giving rise to driving force of grain vibrations. The forced grain vibrations modify the electron transport in the device, which is the indirect effect of the ac gate. For small grain vibrations, the transport in the device is, however, affected little by the grain vibrations and then it is attributed to stochastic electron tunneling as in the conventional SETs. On the other hand, for large grain vibrations, electron tunneling occurs coherently 
when the grain approaches the electrodes and electrons are carried by the grain motion (shuttling). The current is proportional to the gate frequency and current noise is extremely suppressed in contrast to the former, which are the characteristics of shuttle current. Thus, the transport in the device remarkably depends on the grain vibrations.

We studied a switching mechanism between the small and large grain vibrations in order to control the transport mechanisms. On the basis of the balance equation of vibration energy, we predicted occurrence of drastic and hysteretic transitions in the grain vibrations as well as in the transport mechanisms at certain magnitudes of the coupling constant $\kappa$. The numerical simulations demonstrate the hysteretic transitions in the grain vibrations and in the transport properties, manifesting that the ac gate gives rise to shuttling. On the other hand, it is found that the ac gate at off-resonance frequency restrains shuttling even in the shuttle regime in the absence of the gate, then there is no drastic change in the transport properties. Hence, we conclude that the ac gate can actively switch the transport mechanisms in the NEM-SET between tunneling and shuttling, by tuning the gate frequency and $\kappa$, regardless of the regimes in the absence of the gate. Thus, the grain dynamics and transport properties are controllable by the ac gate and then the findings of this work will provide a different mechanism for device applications such as sensors, switching devices, and so on in nanoelectronics.

Finally we propose a feasible NEM-SET that shows the shuttle instability. Bates et al. ${ }^{17}$ fabricated a nanowire made of gold spheres $15 \mathrm{~nm}$ in diameter, which are linked to each other and to electrodes by biomolecules such as DNAs and RNAs. They found that the transport in the nanowire is affected by grain vibrations. We consider a NEM-SET using the gold sphere, which is fabricated in the same method and estimate the capacitance and charging energy as $C$ $=4 \pi \varepsilon_{0} \quad R=0.834 \mathrm{aF}$, where $\varepsilon_{0}$ is the dielectric constant and $\frac{e^{2}}{2 C}=1.53 \times 10^{-20} \mathrm{~J}$. In the estimation, we ignore the effects from the substrate or biomolecules on the effective dielectric constant and put the effective dielectric constant to be 1 . We set the bias voltage as $V=\frac{2 \mathcal{E}}{e}=0.19 \mathrm{~V}$ and estimate $\lambda \approx 0.09 \mathrm{~nm}$ from the work function of gold $(5.1 \mathrm{eV})$. Considering the shuttle instability induced by the ac gate, we put $\kappa_{2}=0.85$ provided that $\frac{\gamma}{\omega_{0}}=0.1$. Assuming the gap between the electrodes to be $d=4 R$, the stiffness constant of the linkage becomes, from Eq. (9),

$$
m \omega_{0}^{2}=\frac{\mathrm{eV}}{\kappa_{2} d \lambda}=0.013 \mathrm{~N} \mathrm{~m}^{-2} .
$$

In contrast to the electronic characteristics, the dynamics of the grain depends on the mechanical properties of the material and structure of the linkage between the grain and electrodes. The strength of the linkage will be adjusted to meet the strength (56) by suitably choosing the material and by configuring the linkages. Using the mass density of gold $\left(\rho=19.3 \times 10^{3} \mathrm{~kg} \mathrm{~m}^{-3}\right), \omega_{0}$ reads

$$
\omega_{0}=0.623 \times 10^{9} \mathrm{rad} \mathrm{s}^{-1} \text {. }
$$

The natural frequency is in the subgigahertz region and it is experimentally possible to tune the gate frequency to the natural frequency. Thus, fabrication of the NEM-SET and the induction of the shuttle instability by the ac gate are possible with existing technologies. Experimental observations of the transitions in the grain dynamics as well as in the transport properties are expected.

\section{ACKNOWLEDGMENTS}

This work is supported in part by a grant-in-aid for scientific research from the Ministry of Education, Culture, Sports, Science and Technology of Japan (Grants No. 1965106507 and No. 20246094).

\section{APPENDIX: COEFFICIENTS $S_{n}$ AND $C_{n}$ OF Eq. (43)}

Solving Eqs. (39) and (40), we obtain

$$
\begin{aligned}
& R_{0}=\frac{1}{2} U_{G}^{0} \cos \left(\theta_{1}\right) \sin \left(\omega t+\psi+\theta-\theta_{1}\right), \\
R_{1}= & \frac{1}{4}\left[2 \cos ^{3} \theta_{1} \sin \left(\omega t-\theta_{1}\right)\right. \\
& +\cos ^{2} \theta_{1} \sin \left(\omega t+2 \psi+2 \theta-2 \theta_{1}\right) \\
- & \left.\cos \theta_{1} \cos \theta_{3} \sin \left(3 \omega t+2 \psi+2 \theta-\theta_{1}-\theta_{3}\right)\right] U_{G}^{02} \\
- & \cos \theta_{1} \sin \left(\omega t-\theta_{1}\right) .
\end{aligned}
$$

Putting Eqs. (A1) and (A2) into Eq. (41), $R_{2}$ is also obtained analytically, although it is not shown because the result is redundant and it is hard to express $R_{2}$ in a concise form. The coefficients $S_{n}$ and $C_{n}(n=0,1$, and 2$)$ are derived using the solution of Eqs. (A1) and (A2) and $R_{2}$ as

$$
\begin{aligned}
& S_{0}=\frac{U_{G}^{0}}{2} \cos \theta_{1} \cos \left(\theta-\theta_{1}\right), \\
& C_{0}=\frac{U_{G}^{0}}{2} \cos \theta_{1} \sin \left(\theta-\theta_{1}\right),
\end{aligned}
$$

$$
S_{1}=-\frac{U_{G}^{0^{2}}}{2} \cos ^{2} \theta_{1} \sin \theta \cos \left(\theta-2 \theta_{1}\right)+\frac{1}{2} \sin 2 \theta_{1},
$$

$$
C_{1}=-\frac{U_{G}^{0^{2}}}{2} \cos ^{2} \theta_{1}\left[\sin \theta \sin \left(\theta-2 \theta_{1}\right)+\frac{1}{2}\right]+\frac{1}{2} \cos \left(2 \theta_{1}\right)+\frac{1}{2},
$$




$$
\begin{aligned}
S_{2}= & \frac{U_{G}^{0^{3}}}{64}\left[-\cos \left(3 \theta-6 \theta_{1}\right)-3 \cos \left(3 \theta-4 \theta_{1}\right)-3 \cos \left(3 \theta-2 \theta_{1}\right)-\cos (3 \theta)+2 \cos \left(\theta-6 \theta_{1}\right)+8 \cos \left(\theta-4 \theta_{1}\right)+11 \cos \left(\theta-2 \theta_{1}\right)\right. \\
& \left.+5 \cos (\theta)-2 \cos \left(\theta+2 \theta_{1}\right)-3 \cos \left(\theta+4 \theta_{1}\right)-\cos \left(\theta+6 \theta_{1}\right)+\cos \left(2 \theta_{3}-\theta\right)+2 \cos \left(2 \theta_{1}+2 \theta_{3}-\theta\right)+\cos \left(4 \theta_{1}+2 \theta_{3}-\theta\right)\right] \\
& +\frac{U_{G}^{0}}{16}\left[-4 \cos \left(\theta-4 \theta_{1}\right)-5 \cos \left(\theta-2 \theta_{1}\right)-2 \cos (\theta)+\cos \left(\theta+2 \theta_{1}\right)+2 \cos \left(\theta+4 \theta_{1}\right)\right] \\
C_{2}= & \frac{U_{G}^{0^{3}}}{64}\left[-\sin \left(3 \theta-6 \theta_{1}\right)-3 \sin \left(3 \theta-4 \theta_{1}\right)-3 \sin \left(3 \theta-2 \theta_{1}\right)-\sin (3 \theta)+2 \sin \left(\theta-6 \theta_{1}\right)+10 \sin \left(\theta-4 \theta_{1}\right)+19 \sin \left(\theta-2 \theta_{1}\right)\right. \\
& \left.+19 \sin (\theta)+12 \sin \left(\theta+2 \theta_{1}\right)+5 \sin \left(\theta+4 \theta_{1}\right)+\sin \left(\theta+6 \theta_{1}\right)-\sin \left(2 \theta_{3}-\theta\right)-2 \sin \left(2 \theta_{1}+2 \theta_{3}-\theta\right)-\sin \left(4 \theta_{1}+2 \theta_{3}-\theta\right)\right] \\
& +\frac{U_{G}^{0}}{16}\left[-4 \sin \left(\theta-4 \theta_{1}\right)-7 \sin \left(\theta-2 \theta_{1}\right)-6 \sin (\theta)-5 \sin \left(\theta+2 \theta_{1}\right)-2 \sin \left(\theta+4 \theta_{1}\right)\right]
\end{aligned}
$$

Here we put

$$
\begin{gathered}
\psi=\frac{\pi}{2}, \\
\theta_{n}=\tan ^{-1} \frac{n \omega_{G}}{\gamma} .
\end{gathered}
$$

*nn@eng.hokudai.ac.jp

${ }^{1}$ R. Knobel and A. N. Cleland, Nature (London) 424, 291 (2003).

${ }^{2}$ M. D. LaHaye, O. Buu, B. Camarota, and K. C. Schwab, Science 304, 74 (2004).

${ }^{3}$ A. D. Armour, M. P. Blencowe, and K. C. Schwab, Phys. Rev. Lett. 88, 148301 (2002).

${ }^{4}$ M. P. Blencowe, Phys. Rep. 395, 159 (2004).

${ }^{5}$ S. Savel'ev, X. Hu, and F. Nori, New J. Phys. 8, 105 (2006).

${ }^{6}$ L. Y. Gorelik, A. Isacsson, M. V. Voinova, B. Kasemo, R. I. Shekhter, and M. Jonson, Phys. Rev. Lett. 80, 4526 (1998).

${ }^{7}$ A. Isscason, L. Y. Gorelik, R. I. Shekhter, and M. Jonson, Physica B (Amsterdam) 92, 166801 (2004).

${ }^{8}$ T. Novotny, A. Donarini, and A.-P. Jauho, Phys. Rev. Lett. 90, 256801 (2003).

${ }^{9}$ N. Nishiguchi, Phys. Rev. B 65, 035403 (2001).
${ }^{10}$ A. D. Armour, M. P. Blencowe, and Y. Zhang, Phys. Rev. B 69, 125313 (2004).

${ }^{11}$ F. Pistolesi, Phys. Rev. B 69, 245409 (2004).

${ }^{12}$ C. Flindt, T. Novotný, and A.-P. Jauho, Europhys. Lett. 69, 475 (2005).

${ }^{13}$ D. Fedorets, L. Y. Gorelik, R. I. Shekhter, and M. Jonson, Phys. Rev. Lett. 92, 166801 (2004).

${ }^{14}$ B. Elattari and S. A. Gurvitz, Phys. Lett. A 292, 289 (2002).

${ }^{15}$ N. G. Van Kampen, Stochastic Processes in Physics and Chemistry (North-Holland, Amsterdam, 2007), Chap. VIII, p. 215.

${ }^{16}$ H. Park, A. K. L. Lim, and A. P. Alivisats, Appl. Phys. Lett. 75, 301 (1999).

${ }^{17}$ A. D. Bates, B. P. Callen, J. M. Cooper, R. Cosstick, C. Geary, A. Glidle, L. Jaeger, J. L. Pearson, M. Proupín-Pérez, C. Xu, and D. R. S. Cumming, Nano Lett. 6, 445 (2006). 